\title{
Review of cisplatin and oxaliplatin in current immunogenic and monoclonal antibody treatments
}

\section{Rao Khalid Mehmood}

\author{
Department of Colorectal and General Surgery, University Board Hospital, Rhyl, North Wales, UK
}

\begin{abstract}
Platinum-based chemotherapy agents initially transformed cancer treatment. However their effectiveness peaked as combined regimes showed little additional benefit in trials. New research frontiers developed with the discovery that conventional chemotherapy can induce immunological cell death by recruiting high mobility group box 1 protein through T-cell immunity. Simultaneously monoclonal antibody agents (not effective as monotherapies) showed good results in combination with conventional chemotherapy. Some of these combinations are currently in use and researchers hope to develop regimes which can offer substantial benefits. Several resistance mechanisms against platinum compounds are known, but more knowledge is still needed to gain a full understanding. It seems reasonable therefore to revisit the pharmacology of these agents, which may also lead to identify rational combinations with monoclonal agents providing regimes with less toxicity and better efficacy. This article reviews the pharmacology of cisplatin and oxaliplatin and explores their possible association with monoclonal antibody treatments.
\end{abstract}

\section{Introduction}

Cisplatin is a common and effective cancer drug. It has revolutionized the treatment of advanced germ cell tumors which were previously considered highly fatal. ${ }^{1-3}$ Side effects (including peripheral neuropathy) and acquired resistance unfortunately have limited its use $\mathrm{e}^{4}$ and paved the way for the development of new compounds. One of these is oxaliplatin with its 1,2 diaminocyclohexane (DACH) carrier ligand, which does not present the nephrotoxicity of cisplatin and is

Correspondence: Rao Khalid Mehmood, Department of Colorectal and General Surgery, University Board Hospital, Rhyl, North Wales, UK.

E-mail: raodilmeer@yahoo.com

Key words: cisplatin, oxaliplatin, monoclonal antibodies, mechanisms of action, DNA adducts, 1, 2-diaminocyclohexane (DACH).

Received for publication: 5 June 2014.

Accepted for publication: 21 August 2014.

This work is licensed under a Creative Commons Attribution NonCommercial 3.0 License (CC BY-NC 3.0).

(C) Copyright R.K. Mehmood, 2014

Licensee PAGEPress, Italy

Oncology Reviews 2014; 8:256

doi:10.4081/oncol.2014.256 active in some cisplatin-resistant tumors. ${ }^{5,6}$ Combinations of oxaliplatin with other chemotherapeutic agents are currently used in colorectal cancer. However no ideal combination of agents with fewer side effects and broader cytotoxicity has been identified yet and efforts to reduce platinum drug resistance and discover new agents are considered potential areas of future development. The advent of monoclonal antibody drugs (MAD) and their combination with conventional agents have opened new research frontiers.

MADs, such as cetuximab, trastuzumab and bevacizumab, are used to treat several cancers including colorectal, breast and lung cancers ${ }^{7,8}$ by inhibiting key proteins associated with tumor development. Bevacizumab targets and blocks the vascular endothelial growth factor (VEGF) involved in angiogenesis. Blocking VEGF stops vascular endothelial cell proliferation resulting in depleted oxygen and nutrient supplies, therefore inhibiting tumor growth. ${ }^{7,9}$ However these agents still cause toxicity and resistance. ${ }^{10,11}$

Conventional chemotherapy can cause immunological cell death by triggering T-cell induced immunity via the recruitment of high mobility group box 1 (HMGB1) protein, which may lead to more effective cancer treatments. Combining chemotherapy with antibodies may help improve the cytotoxicity profile and reduce resistance and toxicity. In this article the pharmacology of cisplatin and oxaliplatin is revisited in order to gain a better understanding of their mechanisms of immunogenic induced cell death and their potential synergy with monoclonal antibodies.

\section{Cisplatin}

Cisplatin is a heavy metal complex containing a central atom of platinum surrounded by two chloride molecules and two ammonia molecules in the cis position (Figure 1). It is soluble in water or saline. ${ }^{12}$

Chloride atoms of cisplatin are displaced in a chemical reaction by nucleophiles, such as water or sulfhydryl groups, rather than enzyme catalyzed metabolism. Cisplatin reversibly binds to plasma proteins, as typically happens in normal drug protein interactions. The platinum component of cisplatin irreversibly binds to plasma proteins, including albumin, transferrin and gamma globulin. ${ }^{12}$ Three hours after a bolus injection, $90 \%$ of plasma platinum is still protein-bound. The complexes formed by albumin and platinum molecules do not dissociate to a significant extent and are eliminated slowly with a minimum half-life of five days. ${ }^{12}$

\section{Cisplatin effect on DNA}

Cisplatin exerts its cytotoxic effect by binding genomic DNA (gDNA) in the cell nucleus. As a result, DNA replication and transcription become irrelevant, thus leading to cell death. . $^{13,14}$

Cisplatin undergoes hydrolysis within the cell producing a highly reactive charged platinum complex $\left[\mathrm{Pt}\left(\mathrm{NH}_{3}\right)_{2} \mathrm{ClH}_{2} \mathrm{O}\right]^{+}$. After further hydrolysis, this complex binds to DNA bases through the N7 atom 
(preferably guanine). This DNA cross-linkage mechanism interferes with cell division and replication. The damaged DNA initiates repair mechanisms, which, if unsuccessful, trigger apoptosis. ${ }^{14,15}$

Cisplatin forms structurally different adducts with DNA. Initially mono-functional adducts are formed, then a further reaction leads to produce DNA intra-strand or inter-strand links. ${ }^{16} 1,2-\mathrm{d}(\mathrm{GpG})$ intrastrands make around $60-65 \%$ of cisplatin DNA adducts, while the remaining $20-25 \%$ consists of 1,2-d(ApG) intra-strands. 1,3 intrastrands account for a small percentage. ${ }^{17}$ Cisplatin forming adducts with mitochondrial DNA and inducing DNA protein cross-links have also been reported. ${ }^{18}$

Each cisplatin adduct unwinds the DNA helix to different degrees. For example 1,2-d(GpG) and 1,2-d(ApG) intra-strands unwind DNA by $13^{\circ}$, while the $1,3-\mathrm{d}(\mathrm{GpXpG})$ intra-strand unwinds DNA by $23^{\circ}$. Despite these differences, their ability to bend the DNA helix remains unchanged $\left(32-35^{\circ}\right) .{ }^{19}$ These combined processes cause irreparable damage resulting in cell death. Debate surrounding which of these mechanisms is the predominant factor in cancer cell death continues, however 1,2-intra-strand DNA adducts are thought to play the major role in cytotoxicity. Explanations include the inability of transplatin to form these kinds of adducts ${ }^{19}$ and the difficulty in removing them by nucleotide excision repair (NER). ${ }^{20,21}$

1,2-d(GpG) or 1,2-d(ApG) adducts demonstrate the highest affinity for the HMGB1 protein. It is postulated that certain HMGB1 proteins may participate in the cellular processing of 1,2 intra-strands formed by cisplatin leading to increased cytotoxicity. ${ }^{22}$ However, the importance of other minor adducts should not be overlooked, when describing the overall cytotoxicity profile of cisplatin. ${ }^{22}$ Comparatively oxaliplatin adducts binds HMGB1 less avidly than cisplatin adducts. ${ }^{23}$.

Cisplatin forms adducts in histone deplete mitochondrial DNA (mtDNA). ${ }^{14,24,25}$ Mitochondria are unable to perform NER, a major pathway for removing cisplatin adducts ${ }^{26}$ and therefore may be important contributors to cisplatins toxicity.

Before cisplatin binds to genomic or mitochondrial DNA, the loss of a chloride group is needed. The higher chloride concentration in extracellular fluids impedes the formation of mono and diaquo cis-Pt(II) species in which one or both chloride groups are replaced by water molecules. ${ }^{14}$ In contrast the low intracellular chloride concentration results in effective hydrolysis of cisplatin adducts and both chloride leaving groups are replaced by water molecules resulting in the formation of the diaquo compound $\mathrm{Pt}(\mathrm{H} 2 \mathrm{O}) 2$ (NH3)2]2+. The two water molecules it contains increase its reactivity with nucleophilic centers of biomolecules. ${ }^{14,27}$

\section{Oxaliplatin effect on DNA}

New platinum drugs were developed to provide better cytotoxicity and fewer side effects than cisplatin. Carboplatin subsequently replaced it in many regimens followed by the introduction of nedaplatin and oxaliplatin. 0xaliplatin showed no cross-resistance with cisplatin and did not exhibit significant nephrotoxicity. Ototoxicity is an unwanted effect of oxaliplatin in addition to sensory and motor neuropathy. ${ }^{28}$

Oxaliplatin is an organoplatinum structure in which the platinum atom is complexed with DACH and with an oxalate ligand as leaving group (Figure 2). A leaving group (or labile atom) is an atom or group of atoms displaced from the stable component taking with itself the bonding electrons. Oxaliplatin undergoes non enzymatic conversion in physiological solutions into active derivatives via displacement of the labile oxalate ligand. Several transient reactive species are formed including monoaquo and diaquo DACH platinum, which covalently bind with macromolecules. Only monoadducts are formed initially, but eventually oxaliplatin attaches simultaneously to two nucleotide bases resulting in DNA cross-links. ${ }^{28,29}$ These cross-links are formed between the $\mathrm{N} 7$ positions of two adjacent guanines (GG), adjacent adenine gua- nines (AG) and guanines separated by an intervening nucleotide (GNG). They inhibit DNA replication and transcription. Oxaliplatin cytotoxicity is cell cycle non-specific. ${ }^{30}$

The precise mechanism of action of oxaliplatin is unclear and largely extrapolated from the understanding of cisplatin and other DACH compounds. ${ }^{5}$ Both cisplatin and oxaliplatin are DNA alkylating agents forming platinated intra-strand and inter-strand cross-links. ${ }^{31}$ Intra strand links contribute significantly to cisplatin cytotoxicity, but they seem less important in relation to oxaliplatin. ${ }^{32}$ The DACH side chain of oxaliplatin is thought to enhance cytotoxicity and abolish cross-resistance between oxaliplatin and other platinum compounds.

The cytotoxicity of platinum drugs is the result of adducts stopping DNA synthesis and repair. Lower numbers of oxaliplatin adducts are required to be more effective than cisplatin, suggesting that other mechanisms are involved in cell death. ${ }^{28}$

Synergism has been demonstrated between oxaliplatin and 5-fluorouracil. Anti-proliferative properties of oxaliplatin combined with 5fluorouracil increased in vitro and in vivo more than either compound alone in several cancer models, including colon cancer, breast cancer and leukemia. ${ }^{30}$

Evidence suggests that DNA adducts are not the sole mechanism of platinum drug cytotoxicity. Oxaliplatin acts in leukemia cell cultures by interfering with RNA and bonds with sulfhydryl groups of cellular proteins inactivating them and impairing the cell function. ${ }^{33}$

The DACH ligand of Oxaliplatin is bulkier and more water soluble

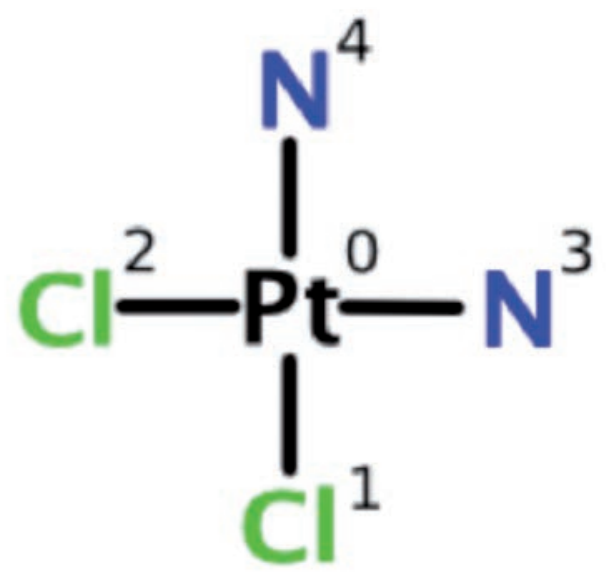

Figure 1. Chemical structure of cisplatin.

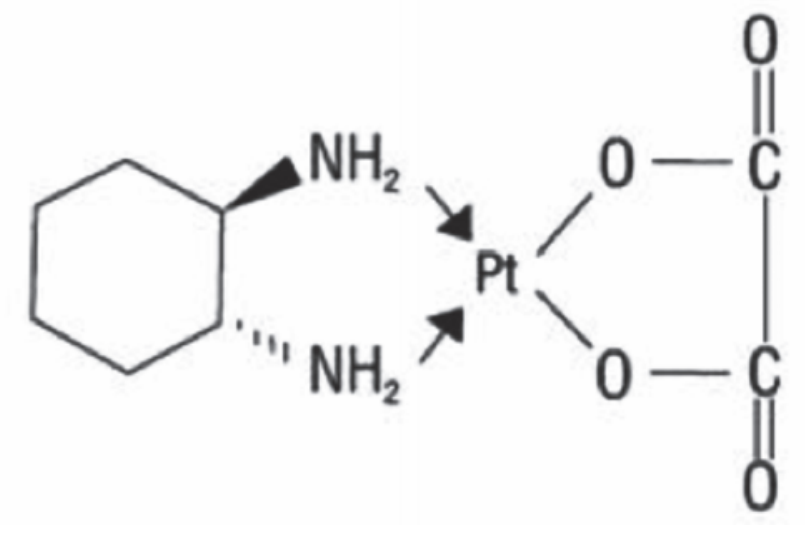

Figure 2. Chemical structure of oxaliplatin. 
than the amino group of cisplatin or carboplatin. This results in greater DNA deformation by adducts which may explain the greater cytotoxicity of oxaliplatin. ${ }^{34}$ The DACH ligand also prevents the mismatch repair complex (MMR) from binding oxaliplatin. ${ }^{3}$ The covalent binding of DNA repair enzymes with oxaliplatin impairs their function ${ }^{33}$ and, if the DNA damage is substantial and irreparable, it may lead to apoptotic pathways and cell death. ${ }^{32}$

\section{Cisplatin and oxaliplatin general mechanisms of action}

\section{Nucleotide excision repair}

The sophisticated NER system repairs DNA lesions inflicted by endogenous or exogenous sources to restore its normal structure. ${ }^{35}$ NER can be of two types, i.e. global genomic NER (GG-NER) and transcription coupled NER (TC-NER), depending on their mode of identifying DNA damage. Cisplatin-induced DNA lesions are mainly repaired by the TCNER pathway. There seems to be no significant difference in the repair of 1,2-d(G* $\left.\mathrm{pG}^{*}\right)$-Pt adducts of cisplatin and oxaliplatin origin. ${ }^{36}$

\section{Transcription coupled repair}

Transcription coupled repair (TCR) is a subdivision of NER. DNA damage is identified during transcription when RNA polymerases are paused and the repair proteins of TCR are recruited resulting in strandspecific lesion repair. ${ }^{37}$ TCR-deficient cells are more sensitive to cisplatin. ${ }^{38}$ TCR repair mechanisms are not fully understood and their role in processing Pt-DNA damage remains an important research area.

\section{Effects on transcription}

Pt-DNA adducts stop in vitro transcription as confirmed by recent experiments in live cells using luciferase assays. ${ }^{36,39}$ One hypothesis suggested that this may be ascribed to the blockage of RNA elongation by DNA adducts. ${ }^{40}$

\section{Repair of Pt-DNA adducts by other mechanisms}

Studies have identified that cells can bypass the transcription processes in the presence of a functioning NER system in order to repair the platinum DNA adducts. This is also possible in the NER deficient XPF cells. Once the transcription process has recovered, it can also remove platinum adducts. Mismatch repair removes platinum adducts as shown in luciferase assays. ${ }^{36,41}$ However these observations need further investigation. ${ }^{41}$

\section{Protein binding with DNA adducts}

Cisplatin DNA adducts bind tightly and selectively with HMGB1, which influences its mechanism of action. ${ }^{42}$

\section{Cisplatin and oxaliplatin cytotoxic mechanisms of action}

DNA damage can result in cell death or repair and survival. One possible apoptotic pathway is the blockage of RNA polymerases by platinum DNA adducts causing transcription cessation and cell death through p53 dependent and independent pathways. ${ }^{43}$

\section{Envisaging tailored platinum chemotherapy based on Pt-DNA adduct processing}

The extent of transcription blockage by DNA platinum adducts depends on their effect on polymerase II, however it is possible for this to be reversed by NER, which restores transcription. Other mechanisms of DNA repair have been mentioned earlier. The understanding of platinum DNA adduct processing in actual cells may help select a tailored drug for an individual treatment from a global or site-specific modified probe in live cells derived from the cancer tissue. ${ }^{36}$

\section{Excision repair cross complementing 1 and xeroderma pigmentosum $A$}

NER activity is increased in cisplatin-resistant cells which appear to be dependent on excision repair cross complementing 1 (ERCC1) and xeroderma pigmentosum A (XPA) expression. An XPA mutation can prevent NER interaction, thus abolishing the DNA repair response. ${ }^{44}$. Testicular germ cell tumors with low XPA can restore the cisplatin adduct removing ability after increasing its expression. These cells have demonstrated increased residual oxaliplatin DNA adducts with greater cytotoxic effects. ${ }^{45}$

ERCC1 is overexpressed in cisplatin resistant cells in vitro. Arnould et al showed that increased ERCC1 expression correlated with fewer cisplatin DNA adducts and reduced cytotoxicity. ${ }^{46}$ Although ERCC1 levels are predictive of oxaliplatin cytotoxicity in many cell lines, they do not correlated with oxaliplatin DNA adducts. ${ }^{47,48}$

\section{Post replication repair}

As the presence of gaps or discontinuities in DNA can be lethal, repair after replication is a major mechanism of DNA damage tolerance. ${ }^{14,49}$ Enzymes involved in post replication repair (PRR) are able to work during DNA synthesis on the leading strand in the presence of platinum adducts. This therefore demonstrates that they do not absolutely hinder DNA replication. They may however affect replicative enzyme performance and accuracy.

Although PRR takes place primarily during cell replication, cisplatin resistant cell lines show an activity during non-replication, therefore indicating that it may be involved in cisplatin resistance. Enzymes involved in PRR include BRCA2, BRCA1 and polymerases (although it is not yet clear which ones actually play a role). High levels of polymerase have been found in a human colon tumor cell line associated with cellular resistance to oxaliplatin. ${ }^{28,50}$

\section{Mismatch repair}

DNA polymerase accuracy is high, but a small percentage of mismatched bases appear in newly synthesized DNA, thus leading to a mutation, if not corrected. The MMR consists of six different proteins, including hMLH1, hMLH2, hPMS2, hMSH2, hMSH3 and hMSH6. Resistance to cisplatin has been reported with defects in these proteins (most likely hMLH1). ${ }^{28,51}$ MLH1 works as a damage recognition unit, like HMGB consistent with its role in cell cycle regulation and apoptosis. ${ }^{28,52}$ In vitro studies demonstrate that MMR appears insignificant in the oxaliplatin-induced DNA damage repair process, but it works as an essential mechanism in cisplatin and carboplatin adduct repair. This results from differing configurational distortion of oxaliplatin DNA adducts due to its DACH ligand. ${ }^{28}$

\section{Damage recognition proteins}

The replicative bypass repairs damaged DNA. Its specificity is determined by DNA polymerases, MMR and damage recognition proteins (DRP) ${ }^{53}$ Only $5-15 \%$ of sporadic tumors are MMR defective,${ }^{54}$ suggesting that other mechanisms influence the specificity of replicative bypass. DRPs bind to platinum DNA adducts decreasing the replicative bypass either by removing new DNA opposite to these adducts with MMR or by blocking the trans-lesion synthesis beyond the adducts. ${ }^{55}$ More than twenty DRPs bind with varying affinities to cisplatin and oxaliplatin adducts. ${ }^{45,56,57}$

DRPs influence the sensitivity to DNA adducts by blocking NER, ${ }^{56}$ 
sequestering transcription factors or activating signal transduction pathways which lead to cell cycle arrest or apoptosis. ${ }^{58}$ The characterization of DNA repair specificity is important in providing models for understanding how repair pathways influence resistance to platinum drugs. $^{45}$

\section{Apoptosis}

The Bcl-2 family of proteins is key in balancing pro-apoptotic and anti-apoptotic stimuli. Anti-apoptotic proteins include Bcl-2, Bcl-XL and Bcl-w, while pro-apoptotic ones are Bax, Bak and Bok. ${ }^{58}$

DNA damage elicits intracellular and extracellular apoptotic responses mediated by p53, abl, c-myc, Rb and E2F. If anti apoptotic factors do not stop them, the mitochondrial membrane potential is decreased, thus leading to cytochrome $\mathrm{C}$ release, oxidative stress, DNA fragmentation and the activation of caspases resulting in cell death. ${ }^{59}$ Cancer cells with high Bcl-2 expression may be less susceptible to apoptosis by cisplatin. ${ }^{60}$

\section{Protein damage}

Apoptotic stimuli are not limited to DNA damage. Protein interactions with oxaliplatin have not been directly investigated, but platinum drugs have a high affinity to cellular proteins. Due to the resemblance of oxaliplatin and cisplatin, they may have similar mechanisms of inducing apoptosis. The hydrophobic DACH moiety in oxaliplatin may facilitate drug interactions inside hydrophobic pockets of cellular proteins. ${ }^{60,61}$ Cisplatin DNA and protein adducts amount to approximately $10 \%$ and $75-85 \%$ respectively. Reactivity of platinum drugs with protein sulfhydryls is likely to distort sufficiently the redox homeostasis of the cell to trigger apoptosis. Thioredoxin has been implicated in cancer cell resistance to cisplatin. Cisplatin can inactivate thioredoxin and its regenerating enzyme thioredoxin reductase. ${ }^{61}$ Faivre et al. found that this enzyme can also be inhibited by oxaliplatin. ${ }^{31}$

DNA and protein damage together may accelerate apoptosis. ${ }^{31}$ The contribution of protein damage to apoptosis changed the belief that the binding of a DNA reactive drug to proteins is merely a detoxification event. ${ }^{62,63}$

\section{Role of p53}

Tumor suppressor gene p53 is essential for cell growth, but it is present at almost undetectable levels in most cells. ${ }^{64,65}$ It regulates DNA replication, repair and recombination in order to eliminate damage. It responds by up regulating Bax synthesis and down regulating Bcl-2 to control mitochondrial permeability and the progression of apoptosis. It translocates to the mitochondria and is sensitive to the levels of Bcl-2 and Bax that they contain. ${ }^{66}$. Mutation of p53 results in a malignant phenotype which occurs in almost all cancers. ${ }^{67}$ Dominant p53 mutations in ovarian cancer cells are a major contributor to cisplatin resistance. ${ }^{66}$ Faivre et al. demonstrated that $\mathrm{p} 53$ defective cells are not necessarily less sensitive to growth inhibition and apoptosis induction by oxaliplatin. $^{31}$

\section{Immunological mechanisms}

The cause of death in cancer cells may be dependent on immunogenic or non-immunogenic mechanisms. Immunogenic cell death initiates changes on the cell surface and release of mediators eventually resulting in cell death. Dendritic cells are antigen-presenting cells which interact with T-cells. Defects in immunogenic signals or in the immune effectors can result in treatment failure with platinum compounds. ${ }^{34,66}$

Immunogenicity of cisplatin and oxaliplatin are different, despite their similarities in the induction of immunogenic cell death (ICD). Oxaliplatin-treated cells interact with T-cells and prime them for the production of interferon anti-cancer vaccination. ${ }^{34}$ Conversely cisplatin-treated cells do not exhibit this mechanism.

Calreticulin (CRT) is a multifunctional protein located in storage compartments associated with the endoplasmic reticulum. Cancer cells cause production of CRT which prompts macrophages to engulf them, however this is counteracted by the blockade of CRT by CD47. Antibodies blocking CD47 may lead to the development of new treatments in the future. Anti CD47 antibodies in mice models of myeloid leukemia and non-Hodgkins lymphoma were successful in eliminating cancer cells without damage to normal cells. ${ }^{68}$.With the release of CRT, also HMGB1 needs to be also produced to achieve ICD. Cisplatin and oxaliplatin are both equally effective in producing both proteins. ${ }^{69}$ In case they fail to induce signals for CRT or HMGB1 release, cell death will be stopped. ${ }^{70}$ CRT induction may be a vital immunogenic mechanism causing reduced efficacy of cisplatin in colorectal cancer patients. $^{69}$

Evidence indicates a strong immunogenic basis of colorectal cancer. Immunological effector cells, such as CD3+ T-cells, CD45R0+ T-cells and macrophages, reduce tumor progression when infiltrated into colorectal cancer tissue. ${ }^{71}$

Toll-like receptor 4 (TLR4) is a protein encoded by the TLR4 gene. ${ }^{72}$ It detects bacteria and cancer cells and lead to the activation of the innate immune system. Oxaliplatin causes expression of immunogenic signals on colorectal cancer cells prior to apoptosis. This activates the innate immune system and results in T-cell interferon production and interaction with TLR4 of dendritic cells creating a tumor vaccine. Patients with mutant TLR4 genes have demonstrated a decreased response to oxaliplatin in metastatic cancer treatment with poorer disease free survival. ${ }^{34}$ Even loss of a functional TLR4 allele was linked with decreased survival in colorectal cancer patients treated with oxaliplatin. Conversely this study demonstrated that TLR4 alleles should not affect the therapeutic response to cisplatin treatment, but more research is required to validate this finding. ${ }^{34,66}$

\section{Resistance}

Resistance to platinum drugs develops in several ways including the low intracellular availability of the drug, increased detoxification inside the cell or strong repair responses due to induced damage. ${ }^{73,74}$

Although not fully understood, platinum drug cellular uptake is a energy-dependent process combined with an efflux pump. This complex mechanism prevents it from being saturable. ${ }^{74}$ This system of uptake and efflux is thought to be the most common mechanism of resistance to cisplatin and is extrapolated to oxaliplatin. ${ }^{75}$

Another resistance mechanism to cisplatin and oxaliplatin is increased glutathione concentration, which inactivates platinum compounds before DNA damage occurs. Metallothioneins are small cysteine-rich proteins involved in metal detoxification and may play a role as stress proteins in response to platinum complexes. ${ }^{76}$ Once inside the cell, platinum drugs are conjugated to glutathione. Enzymes involved in glutathione activity include glutathione S transferase (GST) and glutathione synthase. Once conjugated, these platinum drugs are released and increase drug resistance. GST is a marker of resistance to cisplatin and plays also a vital role in oxaliplatin resistance. ${ }^{77}$

DNA repair is also related to other mechanisms involving systems such as NER, MMR and PRR. Upregulated enzymes in these systems make repair processes more effective and increase drug resistance. Cells that overexpress ERCC1 are resistant to oxaliplatin. ${ }^{78}$ The combination of oxaliplatin with monoclonal antibodies could prevent or even reverse resistance. In vitro assays demonstrated that cetuximab 
reduces the expression of NER components used to remove platinum DNA adducts. ${ }^{79}$

Evidence is increasing that common gene variants (polymorphisms) may have a substantial role in DNA repair and platinum conjugation. Gene coding is involved in the enzymes responsible for oxaliplatin accumulation, detoxicification and DNA adducts repair which may influence the cell response to oxaliplatin. ${ }^{80}$

Deficiencies in apoptotic machinery are associated with cisplatin resistance. Cancer cells with high Bcl-2 expression are less susceptible to apoptosis by cisplatin. ${ }^{66}$ Gourdier et al. conversely found that the modulation of Bax, Bak and Bcl-XL expression is not involved in oxaliplatin resistance.$^{81}$ It is reasonable to suggest that resistance is a combination of processes, therefore efforts should be made to identify them as well as methods to improve the cytotoxicity profile of these drugs.

\section{Toxicity}

\section{Cisplatin}

\section{Nephrotoxicity}

Cisplatin-induced nephrotoxicity is mainly caused by injury to the renal epithelium resulting in an inflammatory response inducing nuclear and mitochondrial DNA injury and activation of cell death. In animal model, drug induced nephrotoxicity is associated with oxygenfree species which can be avoided by using free radical scavenging agents, such as amifostine. ${ }^{82}$

\section{Neurotoxicity}

Neurotoxicity affecting visual perception and hearing abilities starts soon after treatment commences with cisplatin and can be assessed by using pre-treatment and post-treatment nerve conduction studies. ${ }^{83}$ Cisplatin inhibits non competitively NHE-1, a membrane sodium hydrogen ion transporter ${ }^{83}$ found on peripheral nerve cells of the nerve centers receiving ocular and aural stimuli. This interaction with cisplatin is dose-dependent and reversible and results in hydroelectric imbalances and cytoskeleton alterations. ${ }^{83}$

\section{Myelotoxicity}

Cisplatin may be responsible for profound bone marrow suppression and hemolytic anemia. ${ }^{83}$

\section{Oxaliplatin}

\section{The hematopoietic system}

Oxaliplatin is more myelotoxic than cisplatin and severity is dosedependent. Hemolytic anemia and thrombocytopenia are usually not severe, but neutropenia occurs in around $4 \%$ of patients. ${ }^{84}$

Oxaliplatin may affect bone marrow progenitor cells, as its DNA adducts are found in leukocytes after treatment. ${ }^{85}$ The real impact of this hematological toxicity is undefined, but the amount of oxaliplatin DNA adducts in patient blood cells may be related with the severity of their leucopoenia and thrombocytopenia. ${ }^{86}$

Repeated oxaliplatin infusions may result in hypersensitivity reactions which can lead to hemolytic anemia and secondary immune thrombocytopenia. ${ }^{87}$ Some rare cases of secondary acute leukemia have also been reported. ${ }^{88}$

\section{Neurotoxicity}

Acute or chronic peripheral neuropathy is a common side effect of oxaliplatin. Acute peripheral neuropathy can manifest itself as paresthesia, dysthesia, or allodynia of the extremities, lips and orolaryno- gopharynx during or immediately after treatment. ${ }^{89}$ 0xalate, a metabolite of oxaliplatin, interacts with voltage-gated sodium channels in complex pathways involving calcium chelation, ${ }^{90}$ which may block the conduction pathways resulting in peripheral neuropathy. It mainly involves sensory rather than motor fibers.

Repeated oxaliplatin infusions may culminate in chronic peripheral neuropathy which manifests with decreased distal sensations and proprioception. Grade 3 and 4 neuropathy occurs in 15\% of the patients receiving a cumulative oxaliplatin dose of approximately $800 \mathrm{mg} / \mathrm{m}^{2}{ }^{91}$ Initially this was thought to be the result of a degenerative process of the axons, however it has been postulated that the accumulation of oxaliplatin in the dorsal root ganglia cells results in their atrophy and mitochondrial dysfunction. ${ }^{92}$ Fortunately it is reversible in the majority of the cases. Around 5\% of patients have ongoing symptoms and, like its acute counterpart, sensory fibers are mainly involved. ${ }^{34}$

\section{Discussion}

As insights into molecular cancer biology are increasing, new treatment possibilities and pharmacological combinations providing an effective and less toxic treatment will be developed.

Chemotherapy drugs work by stopping cancer cell division with limited selectivity which results in the disruption of normal cells. ${ }^{93}$ This poor selectivity damages rapidly growing non cancer cells, therefore limiting the efficacy of many chemotherapy regimens that cause poor quality of life and drug tolerance. ${ }^{94}$ It may also have a role in drug resistance. ${ }^{69}$

MADs address this problem of selectivity by specifically acting on cancer cells. Cetuximab binds with the extracellular domain of the epidermal growth factor receptor. ${ }^{95,96}$ Similarly trastuzumab binds with the extracellular domain of human epidermal growth factor receptor $2^{97,98}$ and bevacizumab binds with VEGF. ${ }^{99}$ All these interactions are specific to cancer cells and block the specific actions of the relevant receptor or protein.

Cisplatin and oxaliplatin have proven beneficial in treating testicular and colorectal cancers respectively, but their lack of selectivity results in a poor toxicity profile. Their combination with MADs to increase cancer-specific cytotoxicity and decrease side effects is a way forward for future chemotherapeutic regimens. Combining bevacizumab with FOLFOX (folinic acid, 5 flurourocil and oxaliplatin) or XELOX (capecitabine and oxaliplatin) for metastatic colorectal cancer (CRC) demonstrated good response rates and increased disease-free overall survival. ${ }^{100}$ These effects are however restricted to CRC patients diagnosed with an unmutated KRAS gene in their cancers. ${ }^{34,101}$ It is imperative that the use of MADs with conventional agents is based on rational and scientific combinations. This will result from the understanding of their mechanisms of action to design rational trials. It is therefore valuable to revisit the molecular mechanisms of conventional chemotherapeutic agents which will assist in designing new complementary and synergistic combination regimens for future trials. ${ }^{78}$

Molecular predictive markers are also under investigation and require prospective, hypothesis-driven and randomized clinical trials. Only a few molecular predictors have already entered clinical use. This may change in the near future and the majority of therapeutic decisions will account for genetics. ${ }^{102}$

\section{Conclusions}

Understanding the mechanisms of action and resistance of cisplatin and oxaliplatin will facilitate the design of future clinical trials with 
MAD. These combinations will aim to improve their cytotoxicity profile, reduce toxicities, improve treatment outcomes and result in better tolerability and patient satisfaction.

\section{References}

1. Loehrer PJ, Einhorn LH. Drugs five years later. Cisplatin. Ann Intern Med 1984;100:704-13.

2. Ozols RF. Ovarian cancer, Part II: Treatment. Curr Probl Cancer 1992;16:61-126.

3. Ozols RF, Williams S. Testicular cancer. Curr Probl Cancer 1989;13:285-335.

4. Kavanagh J, Tresukosol D, Edwards C, et al. Carboplatin reintroduction after taxane in patients with platinum-refractory epithelial ovarian cancer. J Clin Oncol 1995;13:1584-88.

5. Misset JL, Bleiberg $\mathrm{H}$, Sutherland W, et al. Oxaliplatin clinical activity: a review. Crit Rev Oncol Haematol 2000;35:75-93.

6. Rixe 0, Ortuzar W, Alvarez M, et al. Oxaliplatin, tetraplatin, cisplatin, and carboplatin: spectrum of activity in drug-resistant cell lines and in the cell lines of the National Cancer Institute's Anticancer Drug Screen panel. Biochem. Pharmacol 1996;52:1855-65.

7. Ji JH, Park SH, Lee J, et al. Prospective phase II study of neoadjuvant FOLFOX6 plus cetuximab in patients with colorectal cancer and unresectable liver-only metastasis. Cancer Chemother Pharmacol 2013;72:223-30.

8. Hurwitz HI, Tebbutt NC, Kabbinavar F, et al. Efficacy and safety of bevacizumab in metastatic colorectal cancer: pooled analysis from seven randomized controlled trials. Oncologist 2013;18:1004-12.

9. Alcindor T, Beauger N. Oxaliplatin: a review in the era of molecularly targeted therapy. Curr Oncol 2011;18:18-25.

10. Jin F, Ji H, Jia C, et al. Synergistic antitumor effects of endostar in combination with oxaliplatin via inhibition of HIF and CXCR4 in the colorectal cell line SW1116. PLoS One 2012;7:e47161.

11. Faivre S, Djelloul S, Raymond E. New paradigms in anticancer therapy: targeting multiple signaling pathways with kinase inhibitors. Semin Oncol 2006;33:407-20.

12. Drugs.com. FDA Professional Drug Information - Cisplatin. Available from: http://www.drugs.com/pro/cisplatin.html; http:/www.accessdata.fda.gov/drugsatfda_docs/label/2010/018057 s079lbl.pdf Accessed: 23/05/2014.

13. Gonzalez VM, Fuertes MA, Alonso C, Perez JM. Is cisplatin-induced cell death always produced by apoptosis? Mol Pharmacol 2001;59:657-63.

14. Fuertes MA, Alonso C, Perez JM. Biochemical modulation of cisplatin mechanisms of action: enhancement of antitumor activity and circumvention of drug resistance. Chem Rev 2002;103:645-62.

15. Yang XL, Wang AH. Structural studies of atom-specific anticancer drugs acting on DNA. Pharmacol Ther 1999;83:181-215.

16. Payet D, Gaucheron F, Sip M, Leng M. Instability of the monofunctional adducts in cis-[Pt(NH3)2(N7-N-methyl-2diazapyrenium) $\mathrm{Cl}](2+)$-modified DNA: rates of cross-linking reactions in cis-platinum-modified DNA. Nucleic Acids Res 1993;21:5846-51.

17. Balconi G, Pang Y, Broggini M, et al. Cis dichlorodiammine platinum induced DNA interstrand cross-links in primary cultures of human ovarian cancer. Br J Cancer 1991;64:288-92.

18. Auge P, Kozelka J. Transformations and recognition of platinumDNA adducts: recent developments. Transition Met Chem 1997;22:91-6.

19. Eastman A, Barry MA. Interaction of trans-diamminedichloroplatinum(II) with DNA: formation of monofunctional adducts and their reaction with glutathione. Biochemistry 1987;26:3303-7.
20. Szymkowski DE, Yarema K, Essigmann JM, et al. An intrastrand $\mathrm{d}(\mathrm{GpG})$ platinum crosslink in duplex M13 DNA is refractory to repair by human cell extracts. Proc Natl Acad Sci USA 1992;89:10772-6.

21. Mu D, Shu DS, Sancar A. Reaction mechanism of human DNA repair excision nuclease. J Biol Chem 1996;271:8285-94.

22. Pil PM, Lippard SJ. Specific binding of chromosomal protein HMG1 to DNA damaged by the anticancer drug cisplatin. Science 1992;256:234-7.

23. Graf N, Lippard SJ. Redox activation of metal-based prodrugs as a strategy for drug delivery. Adv Drug Deliv Rev 2012;64:993-1004.

24. Jamieson ER, Lippard SJ. Structure, recognition, and processing of cisplatin-DNA adducts. Chem Rev 1999;99:2467-98.

25. Todd RC, Lovejoy KS, Lippard SJ. Understanding the effect of carbonate ion on cisplatin binding to DNA. J Am Chem Soc 2007;129:6370-1.

26. Perez RP. Cellular and molecular determinants of cisplatin resistance. Eur J Cancer 1998;34:1535-42.

27. Miller SE. The hydrolysis products of cis-diamine-dichloroplatinum(II). 4. The anation kinetics of cis- $\mathrm{Pt}(\mathrm{X})(\mathrm{NH} 3) 2$ $(\mathrm{OH} 2)+(\mathrm{X}=\mathrm{Cl}, \mathrm{OH})$ with glycine, monohydrogen malonate and chloride. Inorg Chim Acta 1991;187:125-32.

28. Kweekel DM, Gelderblom H, Guchelaar HJ. Pharmacology of oxaliplatin and the use of pharmacogenomics to individualise therapy. Cancer Treat Rev 2005;31:90-105.

29. National Cancer Institute. Oxaliplatin. Available from: http://www.cancer.gov/cancertopics/druginfo/oxaliplatin Accessed: $14 / 05 / 2014$.

30. Sanofi. Eloxatin. Summary of product characteristics. Guildford: Sanofi Winthrop Ltd.; September 1999.

31. Faivre S, Chan D, Salinas R, et al. DNA strand breaks and apoptosis induced by oxaliplatin in cancer cells. Biochem Pharmacol 2003;66:225-37.

32. Woynarowski JM, Faivre S, Herzig MC, et al. Oxaliplatin induced damage of cellular DNA. Mol Pharmacol 2000;58:920-7.

33. Raymond E, Faivre S, Chaney S, et al. Cellular and molecular pharmacology of oxaliplatin. Mol Cancer Ther 2002;1:227-35.

34. Alcindor T, Beauger N. Oxaliplatin: a review in the era of molecularly targeted therapy. Curr Oncol 2011;18:18-25.

35. Lu Y, Luo Z, Bregman DB. RNA polymerase II large subunit is cleaved by caspases during DNA damage-induced apoptosis. Biochem Biophys Res Comm 2002;296:954-61.

36. Ang WH, Myint M, Lippard SJ. Transcription inhibition by platinum DNA cross-links in live mammalian cells. J Am Chem Soc 2010;132:7429-35.

37. Svejstrup JQ. Mechanisms of transcription-coupled DNA repair. Nat Rev Mol Cell Biol 2002;3:21-9.

38. Bulmer JT, Zacal NJ, Rainbow AJ. Human cells deficient in transcription-coupled repair show prolonged activation of the Jun $\mathrm{N}$ terminal kinase and increased sensitivity following cisplatin treatment. Cancer Chemother Pharmacol 2005;56:189-98.

39. Jung Y, Lippard SJ. RNA polymerase II blockage by cisplatin-damaged DNA. Stability and polyubiquitylation of stalled polymerase. J Biol Chem 2006;281:1361-70.

40. Todd RC, Lippard SJ. Inhibition of transcription by platinum antitumor compounds. Metallomics 2009;1:280-91.

41. Hoffmann JS, Pillaire MJ, Lesca C, et al. Fork like support bypass replication of lesions that block DNA synthesis on single-stranded templates. Proc Natl Acad Sci U S A 1996;93:13766-9.

42. Wei M, Cohen SM, Silverman AP, Lippard SJ. Effects of spectator ligands on the specific recognition of intrastrand platinum-DNA cross-links by high mobility group box and TATA-binding proteins. J Biol Chem 2001;276:38774-80.

43. Cline SD. Mitochondrial DNA damage and its consequences for 
mitochondrial gene expression. Biochim Biophys Acta 2012;1819: 979-91.

44. Suo Z, Lippard SJ, Johnson KA. Single d(GpG)/cis-diammineplatinum(II) adduct-induced inhibition of DNA polymerization. Biochem 1999;38:715-26.

45. Köberle B, Masters JR, Hartley JA, Wood RD. Defective repair of cisplatin-induced DNA damage caused by reduced XPA protein in testicular germ cell tumours. Curr Biol 1999;9:273-6.

46. Arnould S, Hennebelle I, Canal P, et al. Cellular determinants of oxaliplatin sensitivity in colon cancer cell lines. Eur J Cancer 2003;39:112-9.

47. Shirota Y, Stoehlmacher J Brabender J, et al. ERCC1 and thymidylate synthase mRNA levels predict survival for colorectal cancer patients receiving combination oxaliplatin and fluorouracil chemotherapy. J Clin Oncol 2001;19:4298-304.

48. Han JJ, Baek SK, Lee JJ, et al. Combination of TRAP1 and ERCC1 expression predicts clinical outcomes in metastatic colorectal cancer treated with oxaliplatin/5-fluorouracil. Cancer Res Treat 2014;46:55-64.

49. Dempke W, Voigt W, Grothey A, et al. Cisplatin resistance and oncogenes - a review. Anticancer Drugs 2000;11:225-36.

50. Hartwig A, Blessing H, Schwerdtle T, Walter I. Modulation of DNA repair processes by arsenic and selenium compounds. Toxicology 2003;193:161-9.

51. Di Francesco AM, Ruggiero A, Riccardi R. Cellular and molecular aspects of drugs of the future: oxaliplatin. Cell Mol Life Sci 2002;59:1914-27.

52. Goldberg RM, Sargent DJ, Morton RF, et al. A randomized controlled trial of fluorouracil plus leucovorin, irinotecan, and oxaliplatin combinations in patients with previously untreated metastatic colorectal cancer. J Clin Oncol 2004;22:23-30.

53. Lange SS, Takata K, Wood RD. DNA polymerases and cancer. Nat Rev Cancer 2011;11: 96-110.

54. Yamada M, O'Regan E, Brown R, Karran P. Selective recognition of a cisplatin-DNA adduct by human mismatch repair proteins. Nucleic Acids Res 1997;25:491-6.

55. Katja C, Zimmermann CB, Green DR. The machinery of programmed cell death. Pharm Ther 2001;92:57-70.

56. Lucas MF, Cabeza de Vaca I, Takahashi R, et al. Atomic level rendering of DNA-drug encounter. Biophys J 2014;106:421-9.

57. Kline CL, El-Deiry WS. Personalizing colon cancer therapeutics: targeting old and new mechanisms of action. Pharmaceuticals (Basel) 2013;6:988-1038.

58. Moll UM, Zaika A. Nuclear and mitochondrial apoptotic pathways of p53 FEBS Lett 2001;493:65-9.

59. Qin LF, Ng IO. Induction of apoptosis by cisplatin and its effect on cell cycle-related proteins and cell cycle changes in hepatoma cells. Cancer Lett 2002;175:27-38.

60. Arner ES, Nakamura H, Sasada T et al. Analysis of the inhibition of mammalian thioredoxin, thioredoxin reductase, and glutaredoxin by cis-diamminedichloroplatinum(II) and its major metabolite, the glutathione-platinum complex. Free Radic Biol Med 2001;31:1170-8.

61. Woynarowska BA, Woynarowski JM. Preferential targeting of apoptosis in tumor Versus normal cells. Biochim Biophys Acta 2002;1587:309-17.

62. Shiragami R, Murata S, Kosugi C, et al. Enhanced antitumor activity of cerulenin combined with oxaliplatin in human colon cancer cells. Int J Oncol 2013;43:431-8.

63. Stordal B, Davey M. Understanding cisplatin resistance using cellular models. IUBMB Life 2007;59:696-9.

64. Vousden AK. Activation of the p53 tumor suppressor protein. Biochim Biophys Acta 2002;1602:47-59.

65. Donahue RJ, Razmara M, Hoek JB, Knudsen TB. Direct influence of the p53 tumor suppressor on mitochondrial biogenesis and function. FASEB J 2001;15:635-44.

66. Tesniere A, Schlemmer F, Boige V, et al. Immunogenic death of colon cancer cells treated with oxaliplatin. Oncogene 2010;29:482-91.

67. Donahue RJ, Razmara M, Hoek JB, Knudsen TB. Direct influence of the p53 tumor suppressor on mitochondrial biogenesis and function. FASEB J 2001;15:635-44.

68. Chao MP, Jaiswal S, Weissmann-Tsukamoto R, et al. Calreticulin is the dominant pro-phagocytic signal on multiple human cancers and is counterbalanced by CD47. Sci Transl Med 2010;2:63ra94.

69. Tesniere A, Apetoh L, Ghiringhelli F, et al. Immunogenic cancer cell death: a key-lock paradigm. Curr Opin Immunol 2008;20:504-11.

70. Herzig MC, Liang $\mathrm{H}$, Woynarowski JM. Irofulven induces apoptosis in breast cancer cells regardless of caspase- 3 status. Breast Cancer Res Treat 2002;71:133-43.

71. Peng RQ, Chen RB, Ding Y, et al. Expression of calreticulin is associated with infiltration of T-cells in stage IIIB colon cancer. World J Gastroenterol 2010;16:2428-34.

72. Rock FL, Hardiman G, Timans JC, et al. A family of human receptors structurally related to Drosophila Toll. Proc Natl Acad Sci U S A 1998;95:588-93.

73. Science.gov. Damage repair pathways. Available from: http://www.science.gov/topicpages/d/damage+repair+pathway.ht ml Accessed: 29/04/2014.

74. Go RS, Adjei AA. Review of the comparative pharmacology and clinical activity of cisplatin and carboplatin. J Clin Oncol 1999;17: 409-22.

75. Chen W, Xiao Y, Liu X, et al. Overcoming cisplatin resistance in chemotherapy by biomineralization. Chem Commun 2013;49: 4932-4.

76. Hector S, Bolanowska-Higdon W, Zdanowicz J, et al. In vitro studies on the mechanisms of oxaliplatin resistance. Cancer Chemother Pharmacol 2001;48:398-406.

77. Huang JX, Li FY, Xiao W, et al. Expression of thymidylate synthase and glutathione-s-transferase $\pi$ in patients with esophageal squamous cell carcinoma. World J Gastroenterol 2009;15:4316-21.

78. Ji JH, Park SH, Lee J, et al. Prospective phase II study of neoadjuvant FOLFOX6 plus cetuximab in patients with colorectal cancer and unresectable liver-only metastasis. Cancer Chemother Pharmacol 2013;72:223-30.

79. Sartorius UA, Krammer PH. Upregulation of Bcl-2 is involved in the mediation of chemotherapy resistance in human small cell lung cancer cell lines. Int J Cancer 2002;97:584-92.

80. Sun Z, Chen J, Aakre J, et al. Genetic variation in glutathione metabolism and DNA repair genes predicts survival of small-cell lung cancer patients. Ann Oncol 2010;21:2011-6.

81. Gourdier I, Del Rio M, Pau B, et al. Drug specific resistance to oxaliplatin is associated with apoptosis defect in a cellular model of colon carcinoma. FEBS Lett 2002;529:232-6.

82. ScienceDaily.com. Elucidating side effects of antineoplastic agent. Available from: http://www.sciencedaily.com/releases/2010/10/ 101005141117.htm Accessed: 7/5/2013.

83. Windsor RE, Strauss SJ, Kallis C, et al. Germline genetic polymorphisms may influence chemotherapy response and disease outcome in osteosarcoma: a pilot study. Cancer 2012;118:1856-67.

84. Pieck AC, Drescher A, Wiesmann KG, et al. Oxaliplatin-DNA adduct formation in white blood cells of cancer patients. $\mathrm{Br} \mathrm{J}$ Cancer 2008;98:1959-65.

85. Veal GJ, Dias C, Price L, et al. Influence of cellular factors and pharmacokinetics on the formation of platinum-DNA adducts in leukocytes of children receiving cisplatin therapy. Clin Cancer Res 2001;7:2205-12.

86. Koutras AK, Makatsoris T, Paliogianni F, et al. Oxaliplatin induced acute-onset thrombocytopenia, hemorrhage and hemolysis. 
Oncology 2004;67:179-82.

87. Carneiro BA, Kaminer L, Eldibany M, et al. Oxaliplatin-related acute myelogenous leukemia. Oncologist 2006;11:261-2.

88. Argyriou AA, Polychronopoulos P, Iconomou G, et al. A review on oxaliplatin-induced peripheral nerve damage. Cancer Treat Rev 2008;34:368-77.

89. Grolleau F, Gamelin L, Boisdron-Celle M, et al. A possible explanation for a neurotoxic effect of the anticancer agent oxaliplatin on neuronal voltage-gated sodium channels. J Neurophysiol 2001;85:2293-7.

90. Maindrault-Goebel F, Louvet C, Andre T, et al. Oxaliplatin added to the simplified bimonthly leucovorin and 5-fluorouracil regimen as second-line therapy for metastatic colorectal cancer (folfox6). Eur J Cancer 1999;35:1338-42.

91. Cavaletti G, Tredici G, Petruccioli MG, et al. Effects of different schedules of oxaliplatin treatment on the peripheral nervous system of the rat. Eur J Cancer 2001;37:2457-63.

92. Zafar S, Marcello J, Wheeler J, et al. Treatment-related toxicity and supportive care in metastatic colorectal cancer. J Support Oncol 2010;8:15-20.

93. Apetoh L, Ghiringhelli F, Tesniere A, et al. The interaction between HMGB1 and TLR4 dictates the outcome of anticancer chemotherapy and radiotherapy. Immunol Rev 2007;220:47-59.

94. Reynolds NA, Wagstaff AJ. Cetuximab: In the treatment of metastatic colorectal cancer. Drugs 2004;64:109-18.

95. Cunningham D, Humblet Y, Siena S, et al. Cetuximab monothera- py and cetuximab plus irinotecan in irinotecan-refractory metastatic colorectal cancer. N Engl J Med 2004;351:337-45.

96. Slamon DJ, Leyland-Jones B, Shak S, et al. Use of chemotherapy plus a monoclonal antibody against HER2 for metastatic breast cancer that overexpresses HER2. N Engl J Med 2001;344:783-92.

97. Nahta R, Hortobagyi GN, Esteva FJ. Growth factor receptors in breast cancer: potential for therapeutic intervention. Oncologist 2003;8:5-17.

98. Hurwitz H, Fehrenbacher L, Novotny W, et al. Bevacizumab plus irinotecan, fluorouracil, and leucovorin for metastatic colorectal cancer. N Engl J Med 2004;350:2335-42.

99. Debiec-Rychter M, Cools J, Dumez H, et al. Mechanisms of resistance to imatinib mesylate in gastrointestinal stromal tumors and activity of the PKC412 inhibitor against imatinib-resistant mutants. Gastroenterology 2005;128:270-9.

100. Browder T, Butterfield CE, Kraling BM, et al. Antiangiogenic scheduling of chemotherapy improves efficacy against experimental drug resistant cancer. Cancer Res 2000;60:1878-86.

101. Huber PE, Bischof M, Jenne J, et al. Trimodal cancer treatment: beneficial effects of combined antiangiogenesis, radiation, and chemotherapy. Cancer Res 2005;65:3643-55.

102. European Commission. Cancer Research - Projects funded under the Sixth Framework Programme (2002-2006). Luxembourg: Office for Official Publications of the European Communities; 2008. Available from: ftp://ftp.cordis.europa.eu/pub/lifescihealth/ docs/cancer_web_081117_en.pdf Accessed: 30/05/2014. 